\title{
Polymethoxy-1-Alkenes Screening of Chlorella and Spirulina Food Supplements Coupled with In Vivo Toxicity Studies
}

\author{
Eliana Henao ${ }^{1}$, Patrick J. Murphy ${ }^{2, *}$, Halina Falfushynska ${ }^{3, *}$, Oksana Horyn ${ }^{3}$, \\ Daniel M. Evans ${ }^{2}$, Piotr Klimaszyk ${ }^{4}$ and Piotr Rzymski 5,* \\ 1 Department of Biology, Universidad del Valle, 100-00 Cali, Colombia; eliana.henao@correounivalle.edu.co \\ 2 School of Natural Sciences, Alun Roberts Building (Chemistry), Bangor University, LL57 2UW Bangor, \\ Wales; chs027@bangor.ac.uk \\ 3 Department of Orthopedagogy and Physical Therapy, Ternopil V. Hnatiuk National Pedagogical \\ University, 46027 Ternopil, Ukraine; horynoi@tnpu.edu.ua \\ 4 Department of Water Protection, Adam Mickiewicz University, 61-614 Poznan, Poland; pklim@amu.edu.pl \\ 5 Department of Environmental Medicine, Poznan University of Medical Sciences, 60-806 Poznan, Poland \\ * Correspondence: p.j.murphy@bangor.ac.uk (P.J.M.); falfushynska@tnpu.edu.ua (H.F.); \\ rzymskipiotrl@ump.edu.pl (P.R.)
}

Received: 10 January 2020; Accepted: 7 February 2020; Published: 10 February 2020

\begin{abstract}
Selected species of cyanobacteria and green algae have been reported to produce lipophilic polymethoxy-1-alkenes (PMAs) which were shown to exhibit in vivo teratogenicity. Considering that information on PMAs in Arthospira sp. (known commercially as Spirulina) and Chlorella sp. cultivated for food supplement production was essentially lacking, the present study screened Chlorella $(n=10)$ and Spirulina $(n=13)$ food supplements registered in the European Union. Mass spectrometry analysis of column fractionated extracts was performed. None of the four variants previously reported in some cyanobacteria and green algae, nor any potentially related structures were detected in the studied samples. Since the isolated lipophilic fractions contained various compounds, they were further screened for in vivo teratogenicity in Danio rerio embryo, and for the potential to induce oxidative stress and genotoxicity in the liver and neurotoxicity in the brain of adult zebrafish. None of the tested food supplements had detectable levels of PMAs or any potentially related structures. No teratogenicity was revealed except for spinal curvature induced by fractions obtained from two Chlorella products. Selected fractions revealed cytotoxicity as indicated by an increased level of reactive oxygen species, catalase activity, lipid peroxidation and increased frequency of DNA strand breaks in hepatic tissue. The majority $(60 \%)$ of Chlorella fractions induced an increase in cholinesterase activity in zebrafish brain homogenate while exposure to $61.5 \%$ of Spirulina fractions was associated with its decrease. The present study confirms that Chlorella and Spirulina food supplements are free of teratogenic PMAs, although the observed in vivo toxicities raise questions regarding the quality of selected products.
\end{abstract}

Keywords: cyanotoxins; polymethoxy-1-alkenes; food supplements; Arthrospira; Spirulina; Chlorella; human health risk assessment; zebrafish teratogenicity; oxidative stress; genotoxicity; neurotoxicity

Key Contribution: The commercial microalgal food supplements were not found to contain teratogenic polymethoxy-1-alkenes; although some in vivo toxicities were demonstrated in the case of selected lipophilic fractions obtained from these products. 


\section{Introduction}

There is increasing interest in microalgal food supplements and other microalgae-based foodstuffs. The majority of these are based on Chlorella sp. and Arthrospira sp. (sold under the commercial name of "spirulina"), with the greatest production in Asia, particularly in China [1]. As evidenced, these products can be a source of proteins, vitamins and selected macro- and microelements, particularly iron [2-4]. A number of clinical trials on the therapeutic value of microalgal food supplements have been performed revealing their antihypertensive, antilipidemic, hypoglycemic and immunomodulatory effects [5-7].

Generally, the consumption of food supplements based on Chlorella or Arthrospira biomass has not been associated with health risks, and both are considered safe and are approved by food regulatory bodies such as the European Food Safety Agency (EFSA) or the Food and Drug Administration (FDA) [1]. However, over the years there have been some reports of an increased content of toxic metals, metalloids and cyanotoxins in selected commercial formulas which are attributed to the poor quality of production process (e.g., contaminated culture media, chemical methods of biomass harvesting, co-occurrence of toxigenic cyanobacteria species) [4,8-10]. The observed adverse effects mostly included mild gastrointestinal events such as diarrhea, nausea, abdominal cramps or vomiting [11,12].

Species belonging to the Arthrospira and Chlorella genera are not known to synthesize any toxic compounds that would cause human health risks. However, to our surprise and to the best of our knowledge, the presence of polymethoxy-1-alkenes (PMAs) in these supplements has never been subjected to study. PMAs are lipophilic compounds initially identified in cyanobacterium Tolypothrix conglutinate, and later shown to be present in various other freshwater microalgae, including cyanobacteria (Aphanizomenon ovalisporum, Raphidiopsis raciborskii, Anabaena sp., Nostoc sp., Microcystis sp., Pseudanabaena sp., Scytonema burmanicum, S. ocellatum, and S. mirabilum) and green algae (Pediastrum sp. and Scenedesmus sp.) [13-18]. As shown recently, the presence of PMAs may be strain-dependent or geographically diversified as no evidence was found for A. gracile or R. raciborskii isolated from lakes in Central Europe [19]. Overall, four PMAs varying in chain length have been identified to date (Figure 1). These compounds were also shown to reveal teratogenicity in a Danio rerio embryo experimental model $[17,18]$. However, the in vivo rodent models did not reveal any adverse effect of Chlorella or Spirulina supplementation on pregnancy outcome [20,21]; the use of the latter in pregnant women was shown by one study to correlate with oligohydramnios [22-24]. All in all, this highlights that exploring the presence of PMAs in commercially available microalgal supplements for human consumption is urgently needed. This is particularly important given the fact that these products are reported to be used by pregnant women [22], and some authors suggest that they may be beneficial during pregnancy and lactation as well as early childhood development due to their nutritional value $[25,26]$.
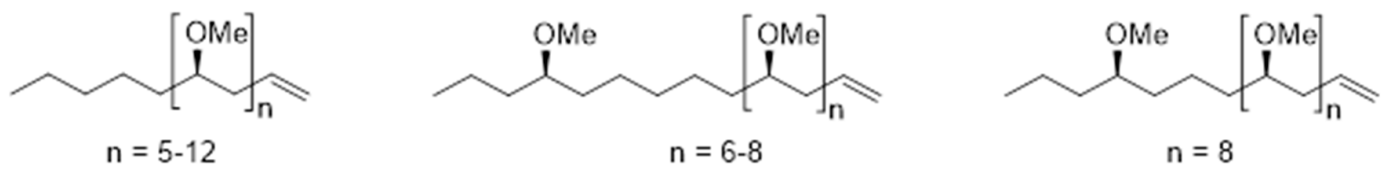

Figure 1. The four polymethoxy-1-alkenes identified so far in selected cyanobacteria and green algae.

In our previous research, we have successfully utilized nuclear magnetic resonance and mass spectrometry to screen PMA content in cyanobacteria A. gracile and $R$. raciborskii isolated from freshwater lakes in Poland. The mass spectrometry is however a method of choice as it is a much more sensitive and was utilized by more recent investigations [17-19]. The aim of this study was to investigate the PMA content in commercial Chlorella and Spirulina food supplements originating from cultivations in North America, Asia and Europe as well as to evaluate the toxicity of isolated lipophilic fractions using a zebrafish Danio rerio experimental model. The toxicological screening was performed because, beside PMAs, microalgae can produce a variety of different lipophilic toxic compounds, including teratogenic retinoic acids and carotenoid glycosides [27,28]. A set of assays 
was employed to evaluate whether the isolated fractions of food supplements can cause teratogenicity in zebrafish embryos as well as induce oxidative stress and genotoxicity in the liver, genotoxicity in peripheral red blood cells and neurotoxicity in the brain of adult zebrafish.

\section{Results}

\subsection{Identification of Polymethoxy-1-Alkenes in Food Supplements}

Ten commercially available Chlorella and thirteen Spirulina food supplements (Table 1) were screened for the content of PMAs using mass spectrometry following extraction of samples in $\mathrm{CHCl}_{3}$, drying under high vacuum and fractionation using silica gel chromatography eluting with a stepwise gradient of ethyl acetate in petrol $(10 \%, 20 \%, 40 \%$ and $100 \%)$. Since no analytical standards were available and no database for PMAs exists, a comparison with literature reporting PMA detection using mass spectrometry was performed $[17,18]$ in all of these fractions. The analysis was targeted towards the identification of known PMAs and also the presence of potential homologous compounds which contain extra $[-\mathrm{CH}=\mathrm{CH}-]_{n},\left[-\mathrm{CH}_{2} \mathrm{CH}(\mathrm{OMe})-\right]_{\mathrm{n}}$ or $\left[-\mathrm{CH}_{2} \mathrm{CH}_{2}-\right]_{n}$ fragments. None of these molecules were apparent in any studied Spirulina and Chlorella food supplement, and analysis of the major peaks did not correlate with these or any potentially related structures.

Table 1. The general characteristics of studied Chlorella $(n=10)$ and Spirulina $(n=13)$ food supplements.

\begin{tabular}{|c|c|c|c|}
\hline Sample Code & $\begin{array}{c}\text { Species Declared } \\
\text { on the Label }\end{array}$ & Country of Origin & Appearance \\
\hline \multicolumn{4}{|c|}{ Chlorella } \\
\hline $\mathrm{C} 1$ & Chlorella sp. & China & Tablets \\
\hline $\mathrm{C} 2$ & Chlorella vulgaris & Taiwan & Tablets \\
\hline $\mathrm{C} 3$ & Chlorella pyrenoidosa & Japan & Tablets \\
\hline $\mathrm{C} 4$ & Chlorella sp. & India & Tablets \\
\hline C5 & Chlorella sp. & China & Powder \\
\hline C6 & Chlorella sp. & China & Powder \\
\hline $\mathrm{C} 7$ & Chlorella pyrenoidosa & China & Powder \\
\hline $\mathrm{C} 8$ & Chlorella vulgaris & China & Powder \\
\hline C9 & Chlorella vulgaris & Portugal & Powder \\
\hline $\mathrm{C} 10$ & Chlorella vulgaris & China & Tablets \\
\hline \multicolumn{4}{|c|}{ Spirulina } \\
\hline S1 & Spirulina sp. & China & Powder \\
\hline S2 & Spirulina sp. & China & Powder \\
\hline S3 & Spirulina sp. & China & Powder \\
\hline S4 & Spirulina platensis & China & Powder \\
\hline S5 & Spirulina sp. & Taiwan & Powder \\
\hline S6 & Spirulina pacifica & USA & Tablets \\
\hline S7 & Arthrospira platensis & China & Powder \\
\hline S8 & Spirulina platensis & China & Tablets \\
\hline S9 & Spirulina sp. & China & Tablets \\
\hline S10 & Spirulina maxima & China & Tablets \\
\hline S11 & Spirulina sp. & China & Tablets \\
\hline S12 & Spirulina sp. & China & Tablets \\
\hline $\mathrm{S} 13$ & Spirulina sp. & India & Powder \\
\hline
\end{tabular}

\subsection{Toxicological Screening}

An extracted lipophilic fraction (100\% ethyl acetate) of each Chlorella (C1-C10) and Spirulina (S1-S13) food supplement was used to test teratogenicity in zebrafish embryo, oxidative stress and genotoxicity in the liver of adult specimens, genotoxicity in peripheral red blood cells and neurotoxicity in the brain. 


\subsubsection{Evaluation of Teratogenicity of Lipophilic Fractions in Zebrafish Embryo}

All control zebrafish embryos (both in Tris-buffer and Tris-buffer $+0.19 \%$ ethanol) met the acceptance criteria of $\geq 90 \%$ survival rate and normal development during the $120 \mathrm{~h}$ of the experiment. The exposure to lipophilic fractions of Chlorella and Spirulina food supplements did not produce any observable effect on survival and fingerprint endpoints, with two exceptions: C1 and C9 in which the scoliosis was induced (Table 2; Table 3). This effect was, however, recorded only after $96 \mathrm{~h}$ of exposure.

Table 2. The effect of lipophilic fractions (100\% ethyl acetate) isolated from Chlorella food supplements (C1-C10) on teratogenicity in zebrafish embryos $(n=6)$ exposed in a static manner for $120 \mathrm{~h}$.

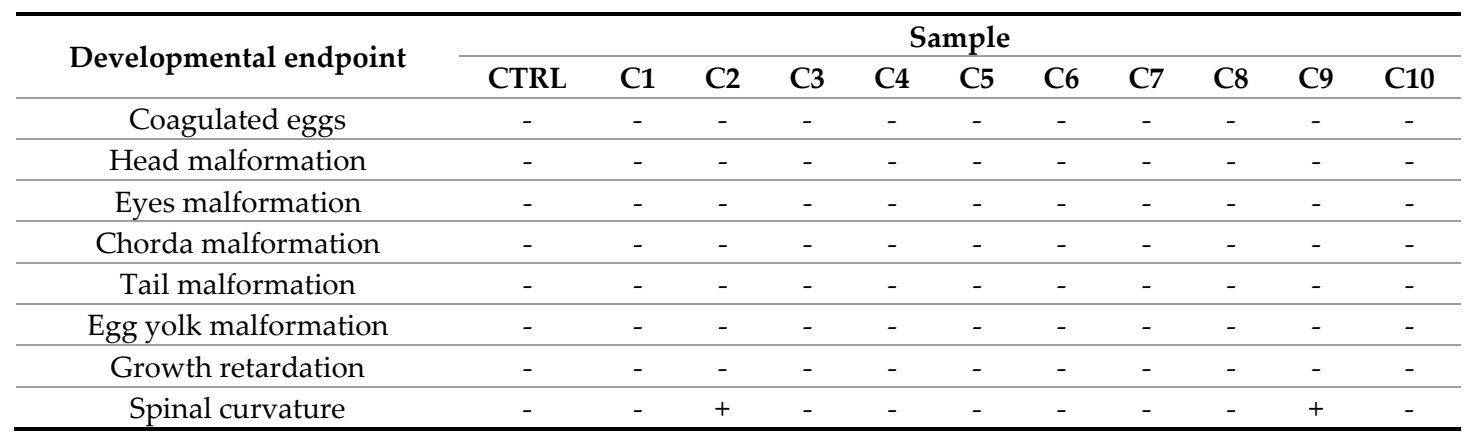

CTRL, control; “-“, normal; “+”, abnormal (reflected in $\geq 50 \%$ of all embryos and/or larvae).

Table 3. The effect of lipophilic fractions ( $100 \%$ ethyl acetate) isolated from Spirulina food supplements $(\mathrm{S} 1-\mathrm{S} 13)$ on teratogenicity in zebrafish embryos $(\mathrm{n}=6)$ exposed in a static manner for $120 \mathrm{~h}$.

\begin{tabular}{|c|c|c|c|c|c|c|c|c|c|c|c|c|c|c|}
\hline \multirow{2}{*}{ Developmental endpoint } & \multicolumn{14}{|c|}{ Sample } \\
\hline & CTRL & S1 & $\mathrm{S} 2$ & S3 & $\mathrm{S} 4$ & S5 & S6 & S7 & S8 & S9 & S10 & S11 & $\mathrm{S} 12$ & $\mathrm{~S} 13$ \\
\hline Coagulated eggs & - & - & - & - & - & - & - & - & - & - & - & - & - & - \\
\hline Head malformation & - & - & - & - & - & - & - & - & - & - & - & - & - & - \\
\hline Eyes malformation & - & - & - & - & - & - & - & - & - & - & - & - & - & - \\
\hline Chorda malformation & - & - & - & - & - & - & - & - & - & - & - & - & - & - \\
\hline Tail malformation & - & - & - & - & - & - & - & - & - & - & - & - & - & - \\
\hline Egg yolk malformation & - & - & - & - & - & - & - & - & - & - & - & - & - & - \\
\hline Growth retardation & - & - & - & - & - & - & - & - & - & - & - & - & - & - \\
\hline Spinal curvature & - & - & - & - & - & - & - & - & - & - & - & - & - & - \\
\hline
\end{tabular}

CTRL-control; “-“, normal; "+”, abnormal (reflected in $\geq 50 \%$ of all embryos and/or larvae).

\subsubsection{Oxidative Stress in Liver of Adult Zebrafish}

The liver tissue collected from adult $D$. rerio revealed increased levels of reactive oxygen species (ROS) and lipid peroxidation (as measured by the content of thiobarbituric acid reactive substances; TBARS) following the exposure of fish to $7 / 10$ and $6 / 10$ fractions of Chlorella supplements, respectively. In comparison, ROS and TBARS were elevated after exposure to 5/13 and 3/13 fractions of Spirulina supplements (Figure 2). Moreover, the exposure to S11-S13 fractions was associated with a decrease in TBARS levels as compared to control. ROS and TBARS were in a positive correlation $(r$ $=0.44, p<0.001)$. Catalase (CAT) activity in hepatic tissue varied from oppression following exposure to C5 and S4 fractions, to two-fold elevation after exposure to the S13 fraction (Figure 2). The majority of fractions had no effect on GST activity, except for C4, C9, S3 and S11 when its decrease was observed, and for $\mathrm{S} 4$ when an increase was recorded. CAT activity revealed a negative correlation with levels of ROS and TBARS ( $r=-0.22, p=0.007$ and $r=-0.30, p=0.001$ correspondingly), whereas no significant correlation for glutathione S-transferase (GST) activity and ROS/TBARS was found ( $p$ $>0.05)$. 
A

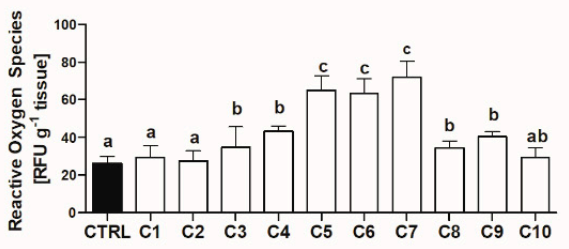

C

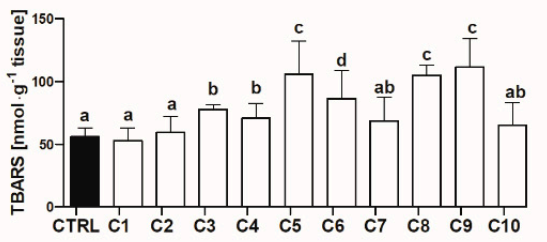

E

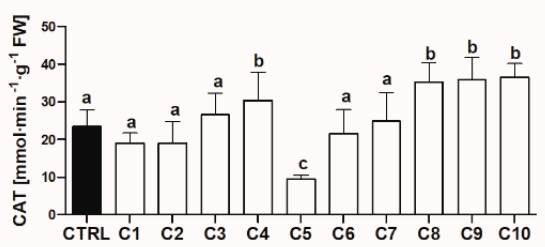

G

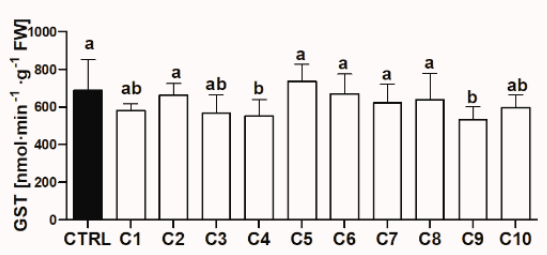

B

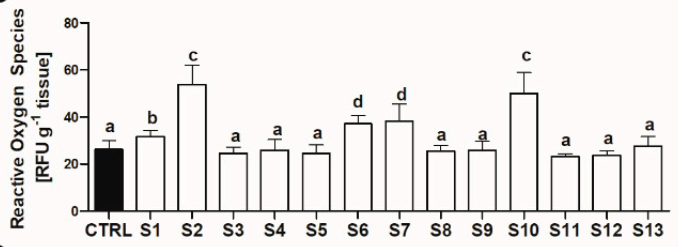

D

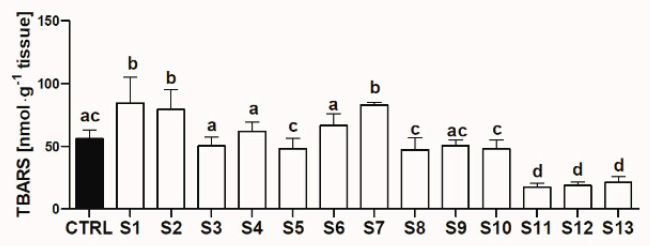

F

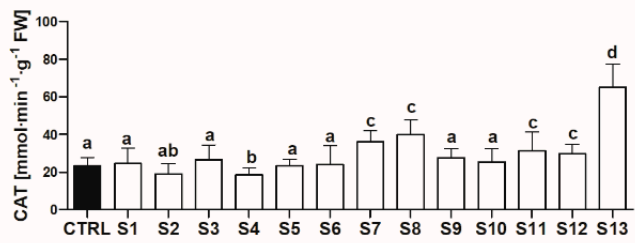

H

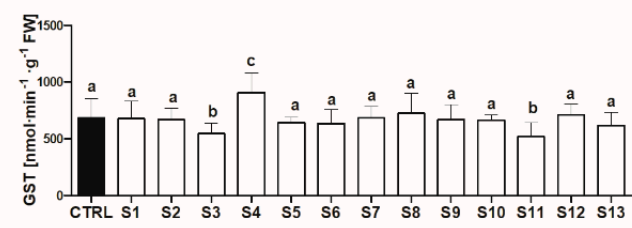

Figure 2. The effect (mean $\pm \mathrm{SD}$ ) of the lipophilic fraction (100\% ethyl acetate) isolated from Chlorella $(\mathbf{A}, \mathbf{C}, \mathbf{E}, \mathbf{G})$ and Spirulina $(\mathbf{B}, \mathbf{D}, \mathbf{F}, \mathbf{H})$ food supplements on reactive oxygen species, lipid peroxidation (TBARS), catalase (CAT) and glutathione S-transferase (GST) activity in hepatic tissue of zebrafish (n =6). Identical superscripts denote no significant differences between treatments (Dunn's test after Kruskal-Wallis ANOVA, $p>0.05)$. CTRL-control.

\subsubsection{Geno and Neurotoxicity Signs in Adult Zebrafish}

Most of tested fractions did not induce DNA strand breaks (DNAsb) in zebrafish liver. Exposure to any Spirulina sample was associated with an increased frequency of micronucleated peripheral red blood cells (Figure 3). Moreover, most of the Chlorella and Spirulina fractions tested induced changes in cholinesterase activity in fish brain with both, a significant decrease (C3, S4, S6, S8-S13) and increase (C5-C10, S1-S3, S5) noted (Figure 3). 

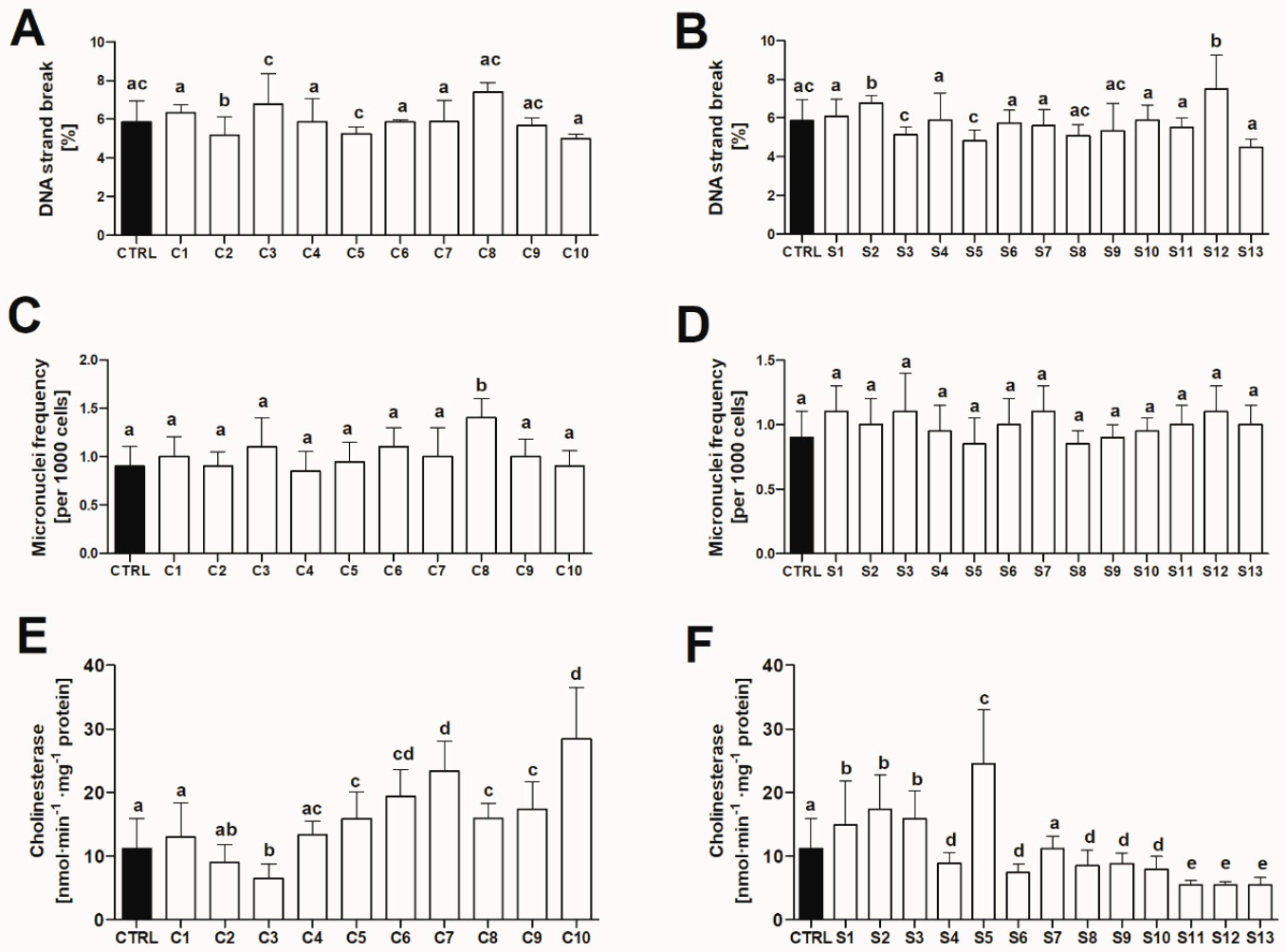

Figure 3. The effect (mean $\pm \mathrm{SD}$ ) of the lipophilic fraction (100\% ethyl acetate) isolated from Chlorella $(\mathbf{A}, \mathbf{C}, \mathbf{E})$ and Spirulina $(\mathbf{B}, \mathbf{D}, \mathbf{E})$ food supplements on DNA strand breaks in hepatic tissue, micronuclei frequency in peripheral red blood cells and cholinesterase activity in the brain of zebrafish $(n=6)$. Identical superscripts denote no significant differences between treatments (Dunn's test after Kruskal-Wallis ANOVA, $p>0.05)$. CTRL-control.

\section{Discussion}

As postulated, ensuring the safety of food supplements based on natural products requires the implementation of analytical screening and the application of toxicity testing $[29,30]$. In the United States and the European Union, the safety of these products remains the responsibility of the manufacturer, while safety assurance is mostly limited to post-marketing surveillance for adverse effects [31]. Regulations have, however, been developed to lessen the possibility of food supplements being contaminated with toxic metals, herbicides or pathogenic microorganisms [31]. Nonetheless not all compounds of potential concern can be subject to random screening. Microalgae are known to produce a wide array of bioactive metabolites, while the precise structures of selected ones remain yet to be elucidated [32-35].

Surprisingly, the content of PMAs, which were found for the first time in cyanobacteria in 1979 [13] and were later proven to be teratogenic [17], has never been investigated for Chlorella or Spirulina food supplements. The broadest screening of these compounds in green algae and cyanobacteria, encompassing a total of 98 strains, was performed by Jaja-Chimedza et al. [18]. However, this study investigated only one isolate of Arthrospira sp. [18] while the production of bioactive metabolites in microalgae, including PMAs in cyanobacteria, has been shown to be geographically diversified and often strain-dependent $[19,36]$. Importantly, such strain-dependency in the production of various compounds, which are present only in small quantities in microalgal biomass, was also documented for strains belonging to the Arthrospira genus [37]. Moreover, one should note that even if PMAs were not produced by any strain of Chlorella and Arthrospira cultivated 
for human consumption, the presence of these compounds in food supplements may potentially originate from culture contamination with other microalgal species. For example, the occurrence of hepatotoxic microcystins in selected food products based on Chlorella and Arthrospira biomass has been linked to the co-occurrence of toxigenic cyanobacteria such as Microcystis [38-42]. The present study rules out the presence of teratogenic PMAs or related structures in both Spirulina and Chlorella food supplements and can confirm consumer safety in this respect.

Nevertheless, one should note that PMAs are not the only teratogenic compounds which could potentially be present in microalgal cells. For example, selected cyanobacteria and green-algae strains have been found to produce retinoic acids and carotenoid glycosides which triggered a diverse set of teratogenic effects in the Danio rerio experimental model $[27,28,43]$. No Arthrospira strains were investigated for the presence of these compounds, while in the case of Chlorella genus, retinoid-like activities were only ruled out for Chlorella kessleri [27]. Therefore, in the present study, we also assessed teratogenicity using the well-established zebrafish embryo assay [44]. As demonstrated, no significant or marked effects on embryo/larvae development were found for the lipophilic fractions obtained from food supplements. The only exception was the spinal curvature induced by two Chlorella samples. The causative agent remains unknown. If one considers that the majority of food supplements from this group did not produce such an effect, it can be postulated that it is likely due to the result of the low quality and potential contamination of selected products. Some Chlorellabased supplements have been shown to contain various polycyclic aromatic hydrocarbons (PAHs) [45,46], and these compounds are known to induce various teratogenic effects [47]. The content of PAHs in microalgal supplements would, however, require a comprehensive screening.

To further explore potential toxicities of lipophilic fractions obtained from the tested food supplements, their potential to induce oxidative stress, genotoxicity and neurotoxicity was also assessed in vivo using adult zebrafish. As demonstrated, selected samples increased the level of ROS, CAT activity, lipid peroxidation (as indicated by the TBARS assay) and increased frequency of DNA strand breaks in hepatic tissue. This indicates that these fractions possessed cytotoxic potency. The cytotoxicity of extracts of Chlorella and Spirulina food supplements were previously demonstrated in vitro in A549 cells, although none of the tested products contained detectable levels of cyanotoxins, such as microcystins, nodularins, saxitoxins, anatoxin-a and cylindrospermopsin [48]. Case reports of hepatotoxicity associated with the use of food supplements based on Chlorella and Spirulina were also reported $[11,49,50]$. All in all, this highlights that the safety of selected microalgal products is questionable. However, one should bear in mind that the fractions of three Spirulina supplements tested in the present study decreased the level of lipid peroxidation as compared to the control which is in line with the hepatoprotective properties of these products reported previously in vitro, in vivo and in clinical trials [51,52].

As demonstrated in the present study, $60 \%$ and $31 \%$ of fractions of Chlorella and Spirulina, respectively, induced an increase in cholinesterase activity in zebrafish brain homogenate. This indicates that these fractions could contain compounds causing a potential loss of cholinergic homeostasis leading to acetylcholine degradation and downregulation of acetylcholine receptors [53]. In turn, the exposure to $61.5 \%$ of Spirulina fractions (and one fraction of Chlorella) was associated with a decrease in cholinesterase activity, which may be associated with increased levels of acetylcholine and overstimulation of cholinergic stimulation [54]. Both an increase and decrease in cholinesterase activity can be associated with adverse effects, although their potential occurrence would require further assessment. There are no known reports of neurotoxicity following the use of Chlorella and Spirulina. However, the findings of the present study suggest that both groups of food supplements may contain lipophilic compounds possessing potential neuromodulatory activities.

\section{Conclusions}

The present study highlights that commercially available microalgal food supplements based on biomass of Spirulina and Chlorella are free of PMAs, while the lipophilic fractions obtained from most of these products do not reveal teratogenicity in a zebrafish model. However, some fractions 
revealed cytotoxicity and neurotoxicity and affected cholinesterase activity. This indicates that the quality and safety of selected microalgal products may be questionable.

\section{Materials and Methods}

\subsection{Food Supplements}

A total of 10 Chlorella-based and 13 Spirulina-based food supplements were randomly purchased from Polish online stores. The inclusion criteria were official registration as a food supplement, tablet or powder form and country of origin declared on the label. The general characteristics of the studied products and the region of origin are summarized in Table 1.

\subsection{Sample Extraction}

The whole batch of each supplement was ground and thoroughly mixed. The powdered samples (10 g) were suspended in $\mathrm{CHCl}_{3}(50 \mathrm{~mL})$ and stirred vigorously for $16 \mathrm{~h}$ then filtered using a sintered filter apparatus. This procedure was repeated a total of three times and the combined extracts dried $\left(\mathrm{MsSO}_{4}\right)$, filtered, the solvent removed under reduced pressure and the residue obtained dried under high vacuum to a constant weight (typically between $5 \%$ to $15 \%$ by mass of extract was obtained; see Supplementary Material for full details). The material obtained was fractioned using silica gel chromatography eluting with a stepwise gradient of $200 \mathrm{~mL}$ each of: $10 \%, 20 \%, 40 \%$ and $100 \%$ ethyl acetate in petrol. Each fraction was collected and evaporated to dryness using a rotary evaporator (Buchi R124 Rotavapor, Flawil, Switzerland) and the ethyl acetate fraction was typically between $0.5 \%$ to $3.0 \%$ by mass of the original supplement (see Supplementary Material for full details).

\subsection{Mass Spectrometry}

To identify PMAs in the obtained fractions a mass spectrometry analysis was performed with an LTQ Orbitrap XL Mass Spectrometer (Thermo Fisher, Altrincham, UK). Initial characterization of samples was made by atmospheric pressure chemical ionization (APCI) mass spectrometry via an Atmospheric Solids Analysis Probe (ASAP) on a Waters Xevo G2-S instrument. A small amount of solid sample was transferred to the tip of a glass capillary, which was then placed within the ASAP source and inserted into the instrument. The vaporizer temperature was increased from $50{ }^{\circ} \mathrm{C}$ to a temperature at which ions were observed and acquired; the discharge current was $4 \mu \mathrm{A}$. Data was processed using vendor MassLynx software. Since no analytical standards were available and no database for these compounds exists, a comparison with literature was performed $[17,18]$.

\subsection{Toxicological Assays}

All zebrafish exposures were conducted at $18 \pm 1^{\circ} \mathrm{C}$. Adult 6-month old zebrafish were obtained from a commercial supplier (Ukraine). The fish underwent an initial acclimation for 7 days and then were randomly distributed to the control, Spirulina (S) and Chlorella (C) exposure groups, with 3 replicate tanks per group, and six specimens per replicate. During acclimation and experimental treatment, $D$. rerio were fed ad libitum with a commercial food. Water was changed every two days. For Spirulina and Chlorella exposures, a static renewal design was used with algae extract addition during each water change. The exposure treatment was continued for 14 days. Clear solution of lipophilic fractions (100\%) of Spirulina and Chlorella in $2 \mathrm{~mL} \mathrm{96 \%} \mathrm{ethanol} \mathrm{was} \mathrm{added} \mathrm{to} \mathrm{the}$ experimental tanks to a nominal concentration of $200 \mu \mathrm{L} \mathrm{L}^{-1}$ by means of dilution of stock solution of lipophilic fractions into 5000 times. Ethical manifestations of Institutional Animal Care and Use Committee protocols (The Institutional Animal Care and Use Committee of the Ternopil V. Hnatiuk National Pedagogical University approved experimental protocols and sampling procedures (approval no. 3/2019, approval date: 3 September 2019). National and international guidelines for the care and use of fisheries were followed. The tissues were sampled according to the Guide for the Care and Use of Laboratory Animals. 
After exposure, the zebrafish were anesthetized by tricaine. Samples of hepatic tissue and peripheral blood from six specimens in each group were prepared individually and kept at $-20{ }^{\circ} \mathrm{C}$ (for less than three days) until analyses. Hepatic and brain tissue was homogenized $(1: 10 \mathrm{w} / \mathrm{v})$ at $4{ }^{\circ} \mathrm{C}$ in $0.1 \mathrm{M} \mathrm{pH} 7.4$ phosphate buffer containing $100 \mathrm{mM} \mathrm{KCl}, 1 \mathrm{mM}$ EDTA and $0.1 \mathrm{mM}$ phenylmethylsulfonylfluoride. The homogenate was centrifuged at $6000 \times g$ for $10 \mathrm{~min}$ at $4{ }^{\circ} \mathrm{C}$. Protein concentration in the supernatant was measured according to Lowry et al. (1951) with bovine serum albumin as a standard. The absorbance was measured with a UV/Vis spectrophotometer, and the fluorescence was measured on the f-max fluorescence microplate reader (Molecular Devices, San Jose, CA, USA).

\subsubsection{Embryotoxicity Testing in Danio Rerio}

The embryo toxicity test was carried out using D. rerio embryos in accordance with the OECD [55]. In brief, adult specimens of wild-type zebrafish (older than 6 months) with high potential to lay fertilized eggs were chosen to spawn, and then, the standard method of breeding was used [55]. Egg production was from female and male spawning groups at a ratio of 1:2, correspondingly. Mating and spawning occurred within $30 \mathrm{~min}$ after turning on the lights in the morning. Egg laying was covered with a plastic mesh for protection against predation. Approx. $30 \mathrm{~min}$ after the light exposure, the egg traps were removed, and the eggs were collected. Following this, the eggs were rinsed in $0.0002 \%$ methylene blue (Sigma-Aldrich, St. Louis, MO, USA), diluted in medium and placed into Petri dishes. Within $2 \mathrm{~h}$ post-fertilization healthy fertilized eggs were selected for the subsequent embryo toxicity test. Zebrafish embryos $(n=6)$ were exposed in a 24-well plate filled with 2 mL Trisbuffer containing $0.4 \mu \mathrm{L}$ of a lipophilic fraction (100\%) of each supplement in $2 \mathrm{~mL} 96 \%$ ethanol at 26 ${ }^{\circ} \mathrm{C}$ with a 12:12-h light/dark cycle. The observed endpoints within $120 \mathrm{~h}$ exposure included mortality, egg coagulation and morphological changes, among which tail and chorda malformation, spinal curvature, otolith and eyes were recorded. The endpoint had to be reflected in $\geq 50 \%$ of all embryos and/or larvae to indicate the teratogenic effect.

\subsubsection{Oxidative Stress Assays}

The rates of reactive oxygen species formation in the liver homogenates in $0.1 \mathrm{M}$ phosphate buffer $\mathrm{pH} 7.4$ were determined using dihydrorhodamine, which is converted by reactive oxygen species to the fluorescent compound rhodamine-123 [56]. The fluorescence signal was registered by an f-max plate-reader [excitation $=485 \mathrm{~nm}$, emission $=538 \mathrm{~nm}$ ], and the rates of reactive oxygen species formation were determined as the difference of the fluorescence at time 0 and after 20 min of incubation and expressed as relative fluorescence unite (RFU) per $1 \mathrm{~g}$ of liver tissue. Lipid peroxidation (LPO) was determined in the supernatant of the liver tissue homogenate $(1: 10 \mathrm{~m} / \mathrm{v})$ in $0.1 \mathrm{M}$ phosphate buffer $\mathrm{pH} 7.4$ using thiobarbituric acid protocol. The level of thiobarbituric acidreactive substances (TBARS) was measured colorimetrically at $532 \mathrm{~nm}$ [57] and calculated using a molar extinction coefficient of $1.56 \times 10^{5} \mathrm{M}^{-1} \cdot \mathrm{cm}^{-1}$. Catalase (CAT) activity was determined in the supernatant of liver tissue homogenate $(1: 10 \mathrm{w}: \mathrm{v})$ in $0.1 \mathrm{M} \mathrm{pH} 7.4$ phosphate buffer by following the decrease in hydrogen peroxide at $240 \mathrm{~nm}$ [58]. CAT activity was determined using the extinction coefficient, $\varepsilon=40 \mathrm{M}^{-1} \cdot \mathrm{cm}^{-1}$ and expressed as $\mathrm{mmol} \times \mathrm{min}^{-1} \times \mathrm{g}^{-1}$ tissue. Glutathione S-transferase (GST) activity was measured in the $D$. rerio liver tissue homogenate $(1: 10 \mathrm{~m} / \mathrm{v})$ using 1 -chloro-2,4dinitrobenzene (CDNB) reduction assay [59]. GST activity was determined as the CDNB absorbance difference over time using an extinction coefficient of $9.6 \mathrm{mM}^{-1} \mathrm{~cm}^{-1}$ and expressed as nmol g-1 tissue.

\subsubsection{Genotoxicity Assays}

To analyze the genotoxicity of lipophilic fractions isolated from Chlorella and Spirulina food supplements, levels of protein-free DNA strand breaks in zebrafish liver tissue homogenate were determined by the alkaline DNA precipitation assay [60] based on using Hoechst 33342 dye in the presence of $4 \mathrm{mM}$ sodium cholate [61]. The fluorescence signal was detected by an $f$-max fluorescence plate-reader $($ excitation $=360 \mathrm{~nm}$, emission $=450 \mathrm{~nm}$ ). The amount of protein-free DNA strand breaks 
was referred to the concentration of the protein in the sample. The genotoxic effect of model exposures was assessed as the micronucleated erythrocytes in the peripheral blood of adult $D$. rerio using Giemsa stain protocol [62]. The frequency of micronuclei was expressed per 1,000 cells.

\subsubsection{Neurotoxicity Assay}

Cholinesterase (ChE, EC 3.1.1.7) activity was determined in the zebrafish brain as acetylthiocholine-cleaving ChE activity according to the colorimetric method of Ellman et al. [63]. ChE activity was calculated using a molar extinction coefficient of $13.6 \cdot 10^{3} \mathrm{M}^{-1} \cdot \mathrm{cm}^{-1}$ and referred to the soluble protein content.

Supplementary Materials: The following are available online at www.mdpi.com/xxx/s1.

Author Contributions: Conceptualization, P.R., P.J.M., H.F. and P.K.; methodology, P.R., P.J.M., D.E., H.F., O.H., P.K. and E.H.; investigation, P.R., P.J.M., D.E., H.F. and O.H.; writing-original draft preparation, P.R., P.J.M., H.F. and E.H.; funding acquisition, P.J.M and H.F. All authors have read and agreed to the published version of the manuscript.

Funding: This research has been partly funded by Beacon+. Beacon+ has been part-funded by the European Regional Development Fund through the Welsh Government. EU Funds: Investing in Wales. Moreover, it was partly supported by the Ministry of Education and Science of Ukraine (project \#MV-2).

Acknowledgments: Thanks are given to the EPSRC National Mass Spectrometry Facility at Swansea and to Rolf Kraehenbuehl, Shon Jones and Bela Paizs for mass spectrometry and MS analysis.

Conflicts of Interest: The authors declare no conflict of interest.

\section{References}

1. García, J.L.; de Vicente, M.; Galán, B. Microalgae, old sustainable food and fashion nutraceuticals. Microb. Biotechnol. 2017, 10, 1017-1024.

2. Buono, S.; Langellotti, A.L.; Martello, A.; Rinna, F.; Fogliano, V. Functional ingredients from microalgae. Food Funct. 2014, 58, 1669-1685.

3. Wells, M.L.; Potin, P.; Craigie, J.S.; Raven, J.A.; Merchant, S.S.; Helliwell, K.E.; Smith, A.G.; Camire, M.E.; Brawley, S.H. Algae as nutritional and functional food sources: Revisiting our understanding. J. Appl. Phycol. 2016, 29, 949-982.

4. Rzymski, P.; Budzulak, J.; Niedzielski, P.; Klimaszyk, P.; Proch, J.; Kozak, L.; Poniedziałek, B. Essential and toxic elements in commercial microalgal food supplements. J. Appl. Phycol. 2019, 31, 3567-3579.

5. Nielsen, C.H.; Balachandran, P.; Christensen, O.; Pugh, N.D.; Tamta, H.; Sufka, K.J.; Wu, X.; Walsted, A.; Schjørring-Thyssen, M.; Enevold, C.; et al. Enhancement of natural killer cell activity in healthy subjects by Immulina ${ }^{\circledR \circledast}$, a Spirulina extract enriched for Braun-type lipoproteins. Planta Med. 2010, 76, 1802-1808.

6. Kim, S.; Kim, J.; Lim, Y.; Kim, Y.J.; Kim, J.Y.; Kwon, O. A dietary cholesterol challenge study to assess Chlorella supplementation in maintaining healthy lipid levels in adults: A double-blinded, randomized, placebo-controlled study. Nutr. J. 2016, 15, 54.

7. Juszkiewicz, A.; Basta, P.; Petriczko, E.; Machaliński, B.; Trzeciak, J.; Łuczkowska, K.; SkarpańskaStejnborn, A. An attempt to induce an immunomodulatory effect in rowers with Spirulina extract. J. Int. Soc. Sports Nutr. 2018, 15, 9.

8. Papazi, A.; Makridis, P.; Divanach, P. Harvesting Chlorella minutissima using cell coagulants. J. Appl. Phycol. 2010, 22, 349-355.

9. Hedegaard, R.; Rokkjær, I.; Sloth, J.J. Total and inorganic arsenic in dietary supplements based on herbs, other botanicals and algae-A possible contributor to inorganic arsenic exposure. Anal. Bioanal. Chem. 2013, 405, 4429-4435.

10. Rzymski, P.; Niedzielski, P.; Kaczmarek, N.; Jurczak, T.; Klimaszyk, P. The multidisciplinary approach to safety and toxicity assessment of microalgae-based food supplements following clinical cases of poisoning. Harmful Algae 2015, 46, 34-42.

11. Marles, R.J.; Barrett, M.L.; Barnes, J.; Chavez, M.L.; Gardiner, P.; Ko, R.; Mahady, G.B.; Low Dog, T.; Sarma, N.D.; Giancaspro, G.I.; et al. United States Pharmacopeia safety evaluation of Spirulina. Crit. Rev. Food Sci. Nutr. 2011, 51, 593-604. 
12. Rzymski, P.; Jaśkiewicz, M. Microalgal food supplements from the perspective of Polish consumers: Patterns of use, adverse events, and beneficial effects. J. Appl. Phycol. 2017, 29, 1841-1850.

13. Mynderse, J.S.; Moore, R.E. Isototactic polymethoxy-1-alkenes from the blue-green alga Tolypothrix conglutinate var. Chlorata. Phytochemistry 1979, 18, 1181-1183.

14. Mori, Y.; Kohchi, Y.; Noguchi, H.; Suzuki, M.; Carmeli, S.; Moore, R.E.; Patterson, G.M.L. Isotactic polymethoxy-1-alkenes from the terrestrial blue-green alga, Scytonema ocellatum: Structure and synthesis. Tetrahedron 1991, 47, 4889-4904.

15. Mori, Y.; Kohchi, Y.; Suzuki, M. Isotactic polymethoxy-1-alkenes from blue-green algae. Synthesis and absolute stereochemistry. J. Org. Chem. 1991, 56, 631-637.

16. Banker, R.; Teltsch, B.; Sukenik, A.; Carmeli, S. 7-Epicylindrospermopsin, a toxic minor metabolite of the cyanobacterium Aphanizomenon ovalisporum from Lake Kinneret, Israel. J. Nat. Prod. 2000, 63, 387-389.

17. Jaja-Chimedza, A.; Gantar, M.; Gibbs, P.D.; Schmale, M.C.; Berry, J.P. Polymethoxy-1-alkenes from Aphanizomenon ovalisporum inhibit vertebrate development in the zebrafish (Danio rerio) embryo model. Mar. Drugs 2012, 10, 2322-2336.

18. Jaja-Chimedza, A.; Saez, C.; Sanchez, K.; Gantar, M.; Berry, J.P. Identification of teratogenic polymethoxy1-alkenes from Cylindrospermopsis raciborskii, and taxonomically diverse freshwater cyanobacteria and green algae. Harmful Algae 2015, 49, 156-161.

19. Rzymski, P.; Evans, D.M.; Murphy, P.J.; Kokociński, M. A study of polymethoxy-1-alkenes in Raphidiopsis (Cylindrospermopsis) raciborskii and Aphanizomenon gracile isolated in Poland. Toxicon 2019, 171, 51-53.

20. Kapoor, R.; Mehta, U. Effect of supplementation of blue green alga (Spirulina) on outcome of pregnancy in rats. Plant Foods Hum. Nutr. 1993, 43, 29-35.

21. Pankaj, P.P. Efficacy of Spirulina platensis in improvement of the reproductive performance and easing teratogenicity in hyperglycemic albino mice. Indian J. Pharmacol. 2015, 47, 430-435.

22. Yusof, J.; Mahdy, Z.A.; Noor, R.M. Use of complementary and alternative medicine in pregnancy and its impact on obstetric outcome. Complement. Therap. Clin. Pract. 2016, 25, 155-163.

23. Nagayama, J.; Noda, K.; Uchikawa, T.; Maruyama, I.; Shimomura, H.; Miyahara, M. Effect of maternal Chlorella supplementation on carotenoid concentration in breast milk at early lactation. Int. J. Food Sci. Nutr. 2014, 65, 573-576.

24. Nakano, S.; Takekoshi, H.; Nakano, M. Chlorella pyrenoidosa supplementation reduces the risk of anemia, proteinuria and edema in pregnant women. Plant Foods Hum. Nutr. 2010, 65, 25-30.

25. Sinha, S.; Patro, N.; Patro, I.K. Maternal protein malnutrition: Current and future perspectives of spirulina supplementation in neuroprotection. Front. Neurosci. 2018, 12, 966.

26. Masuda, K.; Chitundu, M. Multiple Micronutrient Supplementation Using Spirulina platensis during the First 1000 Days is Positively Associated with Development in Children under Five Years: A Follow up of A Randomized Trial in Zambia. Nutrients 2019, 11, 730.

27. Jaja-Chimedza, A.; Sanchez, K.; Gantar, M.; Gibbs, P.; Schmale, M.; Berry, J.P. Carotenoid glycosides from cyanobacteria are teratogenic in the zebrafish (Danio rerio) embryo model. Chemosphere 2017, 174, 478-489.

28. Wu, X.; Jiang, J.; Wan, Y.; Giesy, J.P.; Hu, J. Cyanobacteria blooms produce teratogenic retinoic acids. Proc. Natl. Acad. Sci. USA 2012, 109, 9477-9482.

29. Taylor, D.A. Botanical supplements: Weeding out the health risks. Environ. Health Perspect. 2004, 112, A750A753.

30. van Breemen, R.B.; Fong, H.H.; Farnsworth, N.R. Ensuring the safety of botanical dietary supplements. Am. J. Clin. Nutr. 2008, 87, 509S-513S.

31. Van Breemen, R.B. Development of safe and effective botanical supplements. J. Med. Chem. 2015, 58, 83608372.

32. Fu, W.; Nelson, D.R.; Yi, Z.; Xu, M.; Khraiwesh, B.; Jijakli, K.; Chaiboonchoe, A.; Alzahmi, A.; Al-Khairy, D.; Brynjolfsson, S.; et al. Bioactive compounds from microalgae: Current development and prospects. In Studies in Natural Products Chemistry; Atta ur, R., Ed.; Elsevier: Amsterdam, The Netherlands, 2017; Volume 54, pp. 199-225.

33. Singh, S.; Kate, B.N.; Banerjee, U.C. Bioactive Compounds from Cyanobacteria and Microalgae: An Overview. Crit. Rev. Biotechnol. 2005, 25, 73-95.

34. Bláha, L.; Pavel, B.; Blahoskav, M. Toxins produced in cyanobacterial water blooms-Toxicity and risks. Interdiscip. Toxicol. 2009, 2, 36-41. 
35. Poniedziałek, B.; Rzymski, P.; Kokocinski, M.; Karczewski, J. Toxic potencies of metabolite(s) of noncylindrospermopsin producing Cylindrospermopsis raciborskii isolated from temperate zone in human white cells. Chemosphere 2015, 120, 608-614.

36. Rzymski, P.; Poniedziałek, B. In search of environmental role of cylindrospermopsin: A review on global distribution and ecology of its producers. Water Res. 2014, 66, 320-337.

37. Satora, P.; Barwińska-Sendra, A.; Duda-Chodak, A.; Wajda, Ł. Strain-dependent production of selected bioactive compounds by Cyanobacteria belonging to the Arthrospira genus. J. Appl. Microbiol. 2015, 119, 736-743.

38. Costa, J.A.V.; de Morais, M.G.; Dalcanton, F.; da Cruz Reichert, C.; Durante A.J. Simultaneous cultivation of Spirulina platensis and the toxigenic cyanobacteria Microcystis aeruginosa. Z. Naturforsch C J. Biosci. 2006, 61, 105-110, doi:10.1515/znc-2006-1-219.

39. Jiang, Y. Detection of the hepatotoxic microcystins in 36.660 kinds of cyanobacteria Spirulina food products in China. Food Addit. Contam. Part A Chem. Anal. Control Expo. Risk Assess. 2008, 25, 885-894.

40. Vichi, S.; Lavorini, P.; Funari, E.; Scardala, S.; Testai, E. Contamination by Microcystis and microcystins of blue-green algae food supplements (BGAS) on the Italian market and possible risk for the exposed population. Food Chem. Toxicol. 2012, 50, 4493-4499.

41. Roy-Lachapelle, A.; Solliec, M.; Bouchard, M.F.; Sauve, S. Detection of Cyanotoxins in Algae Dietary Supplements. Toxins 2017, 9, 76.

42. Manali, K.M.; Arunraj, R.; Kumar, T.; Ramya, M. Detection of microcystin producing cyanobacteria in Spirulina dietary supplements using multiplex HRM quantitative PCR. J. Appl. Phycol. 2017, 29, 1279-1286.

43. Jonas, A.; Buranova, V.; Scholz, S.; Fetter, E.; Novakova, K.; Kohoutek, J.; Hilscherova, K. Retinoid-like activity and teratogenic effects of cyanobacterial exudates. Aquat. Toxicol. 2014, 155, 283-290.

44. Truong, L.; Harper, S.; Tanguay, R. Evaluation of Embryotoxicity Using the Zebrafish Model. In Drug Safety Evaluation; Gautier, J.-C., Ed.; Humana Press: New York, NY, USA, 2011; pp. 271-279.

45. Van der Spiegel, M.; Noordam, M.Y.; van der Fels-Klerx, H.J. Safety of novel protein sources (insects, microalgae, seaweed, duckweed and rapeseed) and legislative aspects for application in food and feed production. Compr. Rev. Food Sci. Food Saf. 2013, 12, 662-678.

46. FERA. Occurrence of Polycyclic Aromatic Hydrocarbons in Herbs, Spices, Supplements and Tea Report to Food Standards Agency. $2015 . \quad$ Available online: https://www.food.gov.uk/sites/default/files/media/document/pahs-in-herbs-spices-supplements-and-teafinal-report_0.pdf (accessed on 7 January 2020).

47. Billiard, S.M.; Timme-Laragy, A.R.; Wassenberg, D.M.; Cockman, C.; Di Giulio, R.T. The role of the aryl hydrocarbon receptor pathway in mediating synergistic developmental toxicity of polycyclic aromatic hydrocarbons to zebrafish. Toxicol. Sci. 2006, 92, 526-536.

48. Heussner, A.H.; Mazija, L.; Fastner, J.; Dietrich, D.R. Toxin content and cytotoxicity of algal dietary supplements. Toxicol. Appl. Pharmacol. 2012, 265, 263-271.

49. Iwasa, M.; Yamamoto, M.; Tanaka, Y.; Kaito, M.; Adachi, Y. Spirulina-associated hepatotoxicity. Am. J. Gastroenterol. 2002, 97, 3212-3213.

50. Costa, M.L.; Rodrigues, J.A.; Azevedo, J.; Vasconcelos, V.; Eiras, E.; Campos, M.G. Hepatotoxicity induced by paclitaxel interaction with turmeric in association with a microcystin from a contaminated dietary supplement. Toxicon 2018, 150, 207-211.

51. Mazokopakis, E.E.; Starakis, I.K.; Papadomanolaki, M.G.; Mavroeidi, N.G.; Ganotakis, E.S. The hepatoprotective and hypolipidemic effects of Spirulina (Arthrospira platensis) supplementation in a Cretan population with non-alcoholic fatty liver disease: A prospective pilot study. Ann. Gastroenterol. 2014, 27, 387-394.

52. Gad, A.S.; Khadrawy, Y.A.; El-Nekeety, A.A.; Mohamed, S.R.; Hassan, N.S.; Abdel-Wahhab, M.A. Antioxidant activity and hepatoprotective effects of whey protein and Spirulina in rats. Nutrition 2011, 27, 582-589.

53. Soreq, H.; Seidman, S. Acetylcholinesterase-New roles for an old actor. Nat. Rev. Neurosci. 2011, 2, 294302.

54. Mercey, G.; Verdelet, T.; Renou, J.; Kliachyna, M.; Baati, R.; Nachon, F.; Jean, L.; Renard, P.-Y. Reactivators of Acetylcholinesterase Inhibited by Organophosphorus Nerve Agents. Acc. Chem. Res. 2012, 45, 756-766. 
55. OECD (Organization for Economic Cooperation and Development). OECD Draft Proposal for A New Guideline, 1st Version. In Guideline for the Testing of Chemicals; Fish Embryo Toxicity, FET Test; Paris, France, 2006.

56. Poniedziałek, B.; Rzymski, P.; Karczewski, J. The role of the enzymatic antioxidant system in cylindrospermopsin-induced toxicity in human lymphocytes. Toxicol. In Vitro 2015, 29, 926-932.

57. Ohkawa, H.; Ohishi, N.; Yagi, K. Assay for lipid peroxides in animal tissues by thiobarbituric acid reaction. Anal. Biochem. 1979, 139, 292-298.

58. Aebi, H. Catalase. In Methods of Enzymatic Analysis; Bergmeyer, H.U., Ed.; Verlag Chemie/Academic Press Inc.: Weinheim, Germany; NewYork, NY, USA, 1974; pp. 673-680.

59. Habig, W.H.; Pabst, M.J.; Jacoby, W.B. Glutathione-S-transferases. The first enzymatic step in mercapturic acid formation. J. Biol. Chem. 1974, 249, 7130-7139.

60. Olive, P.L. DNA precipitation assay: A rapid and simple method for detecting DNA damage in mammalian cells. Environ. Mol. Mutagen. 1988, 11, 487-495.

61. Bester, M.J.; Potgieter, H.C.; Vermaak, W.J.H. Cholate and $\mathrm{pH}$ reduce interference by SDS in the determination of DNA with Hoescht. Anal. Biochem. 1994, 223, 299-305.

62. Barsiene, J.; Syvokiene, J.; Bjornstad, A. Induction of micronuclei and other nuclear abnormalities in mussels exposed to bisphenol A, diallyl phthalate and tetrabromodiphenyl ether-47. Aquat. Toxicol. 2006, 78, S105-S108.

63. Ellman, G.L.; Courtney, K.D.; Andres, V., Jr.; Featherstone, R.M. A new and rapid colorimetric determination of acetylcholinesterase activity. Biochem. Pharm. 1961, 7, 88-95.

(C) 2020 by the authors. Licensee MDPI, Basel, Switzerland. This article is an open access article distributed under the terms and conditions of the Creative Commons Attribution (CC BY) license (http://creativecommons.org/licenses/by/4.0/). 